\title{
Intravascular endothelioma (endothelioma in situ, systemic endotheliomatosis)
}

\author{
H. HABER ${ }^{1}$, J. N. HARRIS-JONES, AND A. L. WELLS \\ From the Department of Pathology, St. John's Hospital for Diseases of the Skin, London, and \\ Llanelly Hospital, Llanelly, Carms.
}

SYNOPSIS A woman aged 48 years was found to be suffering from intravascular endothelioma. The initial clinical presentation was the presence of red, painless swellings in the skin of the lower limbs The other clinical features, and the histological changes in skin biopsies, are described ando compared with those in the two previously reported patients. It is suggested that the condition is? analogous to the pre-invasive carcinomatous states.

\section{CASE REPORT}

In August 1960, an apparently healthy woman of 48 years of age noticed the appearance of painless, plumcoloured swellings on the outer aspects of both legs, but dismissed them as being due to insect bites. They had not resolved by October and she sought medical advice. She was treated with antihistaminic drugs, but by January, 1961, the lumps had increased in size and number, and the patient was referred to one of us (J.N.H.-J).

In the past, because of a goitre, her thyroid function had been investigated, with normal results. She had also had a short pyrexial illness characterized by pharyngitis and cervical lymphadenopathy, resembling glandular fever, but agglutination tests for heterophile antibody were negative.

On examination she appeared as a perfectly fit woman whose only symptom was the presence of painless but disfiguring lumps on her legs. She denied significant loss of weight or appetite. The only other abnormal finding was a nodular enlargement of the thyroid gland, without hint of malignancy or of thyrotoxicosis. It was thought that the spleen was just palpable on full inspiration, but the liver was not enlarged nor was there evidence of lymphadenopathy.

The skin lesions were, however, both striking and unusual. These infiltrates were sited over the outer aspects of the leg, thigh, and buttock, both lower limbs being equally, but not symmetrically, involved: there were two larger areas on the trunk, one over the right breast, and the other over the right scapula. The lesions were all essentially of the same type and colour, but varied in diameter from about $1 \mathrm{~cm}$. to $3 \mathrm{~cm}$. The main body of each lesion was of a dusky red hue, edged by a vascular corona of a lighter red which itself faded irregularly into the pink of normal healthy skin. Tortuous vessels coursed through some areas in the deeper layers of the cutis. Most of the lesions were raised and

${ }^{1}$ Dr. Haber died on 7 July, 1962.

Received for publication 25 February, 1964. plaque-like, above the level of surrounding skin, ye $\stackrel{\Phi}{8}$ appearing to have raised the skin, rather than being within the cutis: the skin was slightly puckered where the lesions were attached to its deep surface. A few exhibitedo a slight central depression over which the skin was ap little darker, more atrophic, and showed early exfoliation as if these were older lesions undergoing some degreee of regression.

All tumours were very slightly tender on pressure, an could be moved fractionally in the subcutaneous fat in which most of them appeared to be situated. Apart fromb these indurated areas the skin was normal, and in par ticular there was no evidence of purpura. The exac? nature of these lesions seemed far from clear, except that they were of vascular origin, possibly of the Kapos sarcoma type.

Radiographs of the chest and abdomen, and, becausem of the enlarged thyroid gland, B.M.R. and radio-iodine studies, all gave normal results. The blood count, bones marrow obtained by sternal puncture, and the E.S.R. were normal, apart from a platelet count of 90,000 peê c.mm., which was regarded as a low value.

Biopsy of a typical lesion showed that the tumour was clearly malignant, and appeared to have arisen from vascular tissue. However, in view of the patient's apparene. good health, at her wish she was discharged from hospital

Shortly after her discharge, a further biopsy was pero formed in another hospital. The sections showed changes identical to those seen previously. During this secono admission, in June 1961, her clinical state changed rapidly. She developed a right hemiplegia, and it was suspected that there was a mass in the right loin. Neoo plasm of the right kidney, with metastases in skin an $\bar{\phi}^{2}$ brain, was considered a possibility by those in clinicap charge at the time. Other vascular tumours appeared over the trunk, accompanied by irregular fever, tachy cardia, and generalized oedema of the trunk and lowet⿱ limbs. Pulmonary and urinary infections preceded he? death in August 1961. Despite repeated and determine $\$$ requests for a necropsy, permission was refused. 


\section{PATHOLOGY}

The epidermis in the sections stained with haematoxylin and eosin shows slight and focal hyperkeratosis, but no other abnormality. Conspicuous changes are, however, present in the subepithelial tissues. They involve not only active new formation of thin-walled blood vessels devoid of a muscle coat, but also the occupation of the lumina of these new channels by proliferating tumour cells. They are most marked at the junction of the dermis and subcutaneous fat, whence they extend both into the superficial dermis in the vicinity of the skin appendages, and also deeply into the fat. The newly formed vessels tend to be arranged in clusters, sometimes in the vicinity of small arteries and veins. These clusters occupy the fat in an irregular fashion, enclosing variously shaped groups of fat cells. In the superficial dermis they are present in the vicinity of sweat glands, pilo-sebaceous units, and muscle and nerve fibres. The individual tumour cells are for the most part of uniform size (average $15 \mu$ ). They possess round or oval, slightly indented, and occasionally hyperchromatic nuclei. The cell outlines are indistinct. Mitotic figures are infrequent. Num-

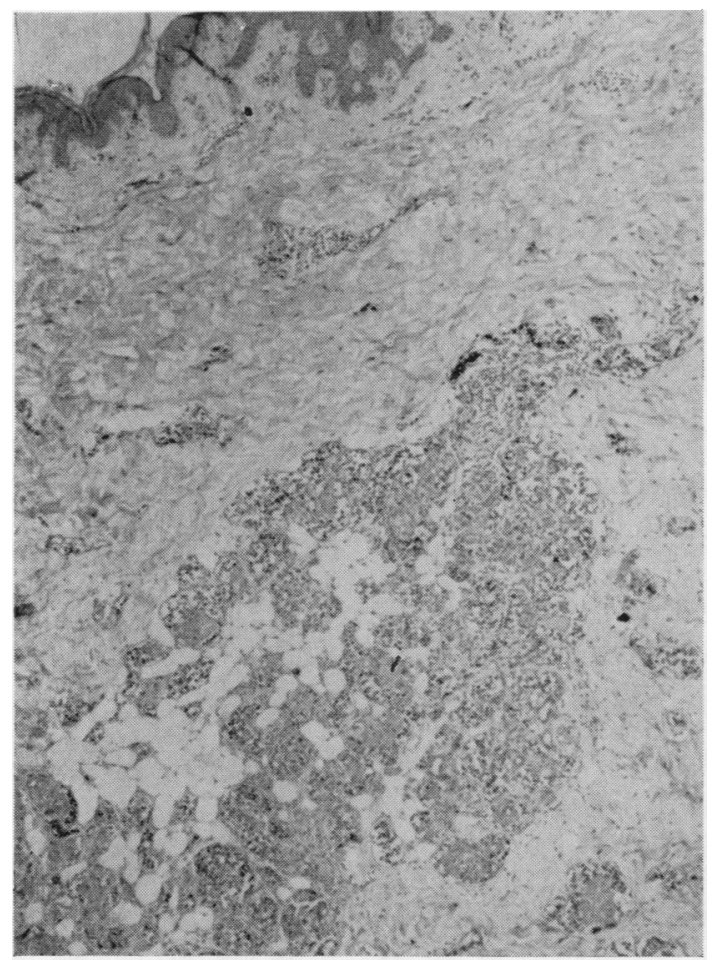

FIG. 1. Edge of lesion showing clusters of affected vessels infiltrating fat. Haematoxylin and eosin $\times 42$. bers of the cells are in various stages of necrosis, and fragmented and pyknotic nuclei can be seen. In the residual spaces, red cells and leucocytes circulate. In many of the affected vessels, hyaline eosinophilic material resembling fibrin is also present. Thus, the vessel lumen may be filled entirely with tumour cells, or partly with tumour cells and partly with fibrin, or exclusively by the latter, in various stages of organization.

The lining of the vascular channels is composed partly of the usual flattened normal endothelial cells, but it is of considerable interest that many tumour cells themselves form the vascular lining. This feature, coupled with the other appearances, leads to a strong presumption that the tumour is to be placed in the reticulo-endothelial group.

Reticulin impregnations show the outlines of the individual vessels, but only occasional fibrils are present inside, except where organization of the fibrin masses is taking place and reticulin is more abundant. Stained by the periodic acid-Schiff technique, the tumour cells give a negative reaction. Elastic stains do not show any features worthy of consideration. In some instances, the presence of the tumour cells is detectable in small arteries and

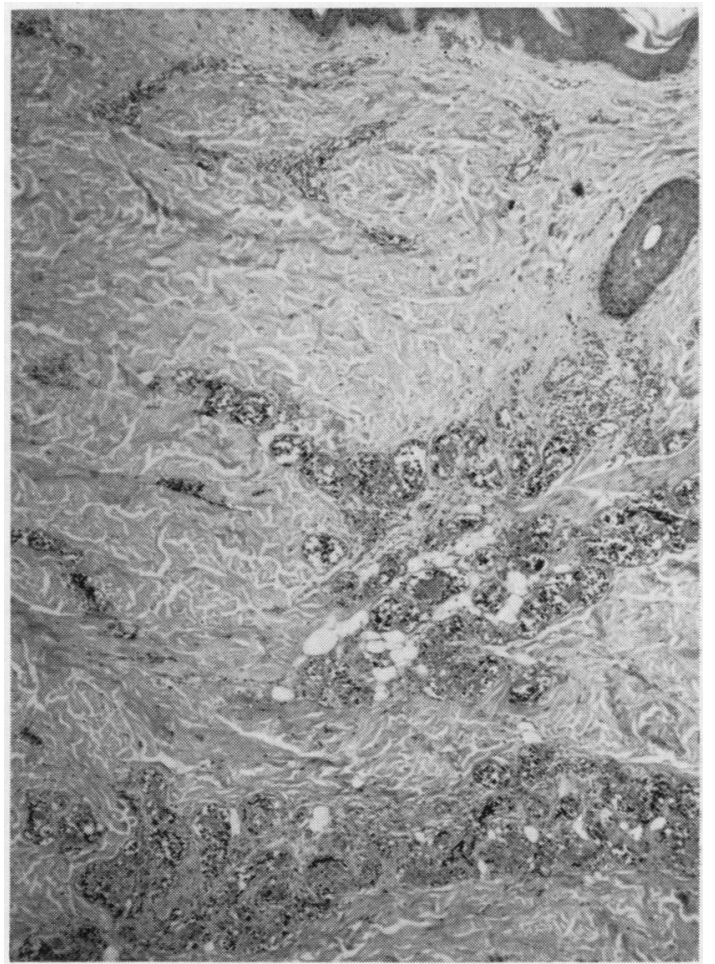

FIG. 2. Similar vessels infiltrating to the vicinity of a hair follicle. Haematoxylin and eosin $\times 42$. 
veins, again apparently acting as part of the lining, but not filling up the lumina in the manner observed in the capillary channels.

A light perivascular mantle of mononuclear cells has developed in relation to some vascular clusters. All the sections examined, however, are free from evidence of spread of the process outside the confines of the vascular spaces. It is probably pertinent that the available biopsies were obtained comparatively early in the evolution of the disease.
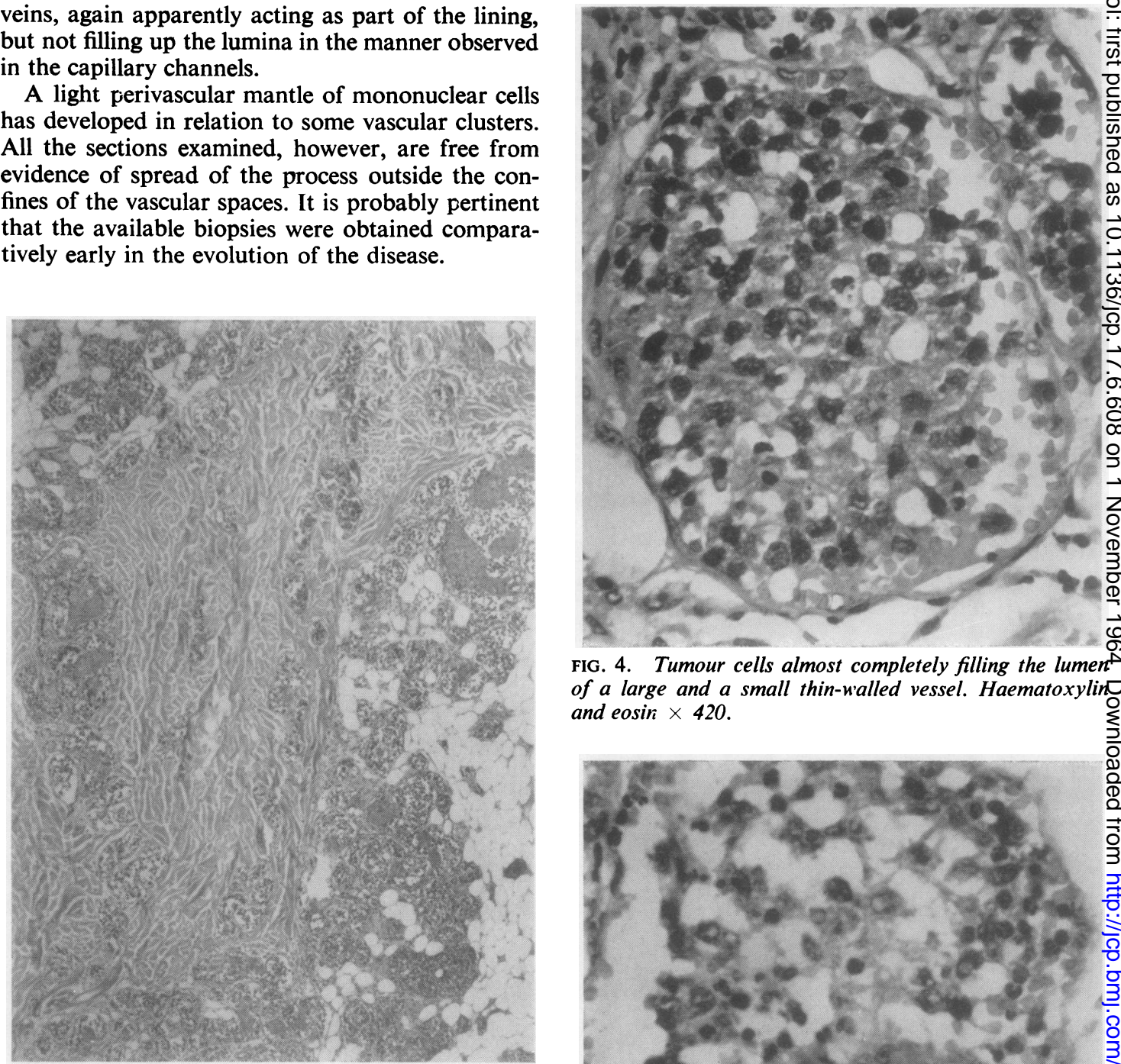

FIG. 4. Tumour cells almost completely filling the lumen ${ }^{\triangleright}$ of a large and a small thin-walled vessel. Haematoxylin and eosin $\times 420$.

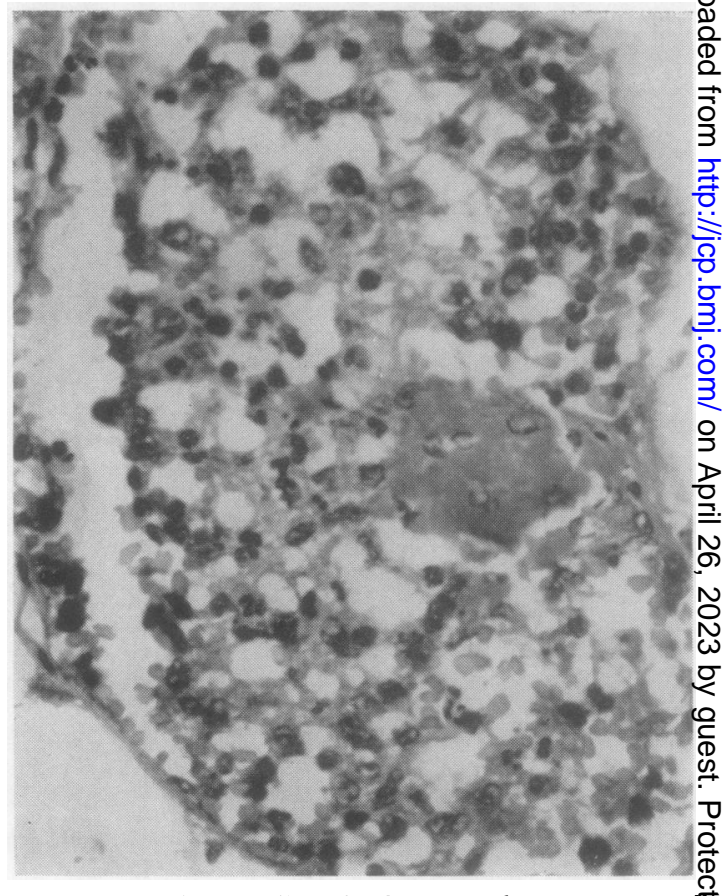

FIG. 3. Tumour in fat and collagen deep in lesion. Haematoxylin and eosin $\times 42$.

\section{DISCUSSION}

The clinical course of this illness followed a definite pattern. It was ushered in by the appearance of painless, dusky red, indurated areas in the skin of the lower limbs. These lesions progressed eventually to involve the limbs and trunk, but throughout the greater part of the illness the patient otherwise remained in good health. Signs of cerebral and abdominal involvement appeared later and were followed by death. The clinical features closely resemble those described by Braverman and Lerner (1961) as having occurred in a woman aged 66 years.

FIG. 5. Similarly affected channel, showing a smal fibrinous area, Haematoxylin and eosin $\times 420$. 
Pfleger and Tappeiner earlier (1959) described the case of a woman aged 31 years, with apparently similar skin lesions, but in whom the disease ran a much more chronic course and who was believed still to be alive. There is a close identity also between the histological findings in each of the previously reported cases and the present patient. The picture of intravascular proliferation of tumour cells, with features strongly suggesting an origin from vascular endothelium, is common to the three cases. That described by Braverman and Lerner showed the additional late feature of extravascular tissue invasion, which they interpreted as a terminal malignant event in the evolution of an otherwise preinvasive process. It is possible that the present case would have exhibitied the same invasive phenomenon had the opportunity been granted to us to make a necropsy study.

Although the morbid anatomical features dictate that the lesion be placed in the broad group of reticulo-endothelial tumours, the apparent absence of involvement of the 'classical' reticulosis sites, i.e., liver, spleen, bone marrow, and lymph nodes, sets it apart from them. Braverman and Lerner draw attention to the presence of the disease process in organs often uninvolved in the 'classical' reticuloses. Moreover, neither would these features suggest the Kaposi lesion or other forms of angiosarcoma. We agree, therefore, with the previous writers that these cases represent a hitherto undescribed clinical and pathological entity. We would also wish to advance a new view, that there is a close analogy between the initial intravascular stage of this process and the pre-invasive growths occurring in squamous and other epithelial surfaces. The latter are known to exhibit great variation in the length of time that may elapse before invasion outside the epithelium takes place, e.g., in the pre-invasive carcinoma of the cervix uteri. Moreover, this particular condition may never become frankly invasive, and the chronicity and possibly benign nature of the case of
Pfleger and Tappeiner may represent a form of vascular disease destined never to spread beyond the confines of the parent vessels. However, the exact fate of this patient is not known.

Equally, there is no doubt that the terminal stages of the patient described by Braverman and Lerner can be regarded as analogous to the invasive phase of other epithelial neoplastic processes referred to previously. One point of difference can, however, be established, on consideration of the comparative effects of the process on the function of the parts involved. Whereas pre-invasive carcinoma in the cervix uteri and other epithelia can exist indefinitely without interference with their function, endothelioma in situ may produce effects by interfering with the blood supply of various tissues without having to exist in the extravascular form.

We are aware that our failure to obtain a necropsy detracts from the value of this communication. However, Tappeiner has examined the histological sections of all three cases, and is of the opinion that they are identical (Braverman, 1963): material from the present case was demonstrated at the International Congress of Dermatology held in September 1962. One of us (A.L.W.) has had the opportunity to examine a histological section from the case of Braverman and Lerner. There can be no reasonable doubt that these cases are all examples of the same unusual syndrome.

The junior authors are grateful to Dr. J. O. Oliver for his willing cooperation in making available papers and material after the death of Dr. Haber. Professor Jethro Gough kindly read the manuscript. We are indebted to Mr. I. H. Davies, F.I.M.L.T., and Mr. H. Fry, F.I.M.L.T., for photographic and technical assistance respectively.

\section{REFERENCES}

Braverman, I. M., and Lerner, A. B. (1961). Arch. Derm., 84, 22. - (1963). Personal communication.

Pfleger, L., and Tappeiner, J. (1959). Hautarzt, 10, 359. 